\title{
Radar signal sorting method in dual airborne-platform cooperative reconnaissance
}

\author{
He Gang ${ }^{1, a}$, Liu Hao ${ }^{1}$, Li xin ${ }^{1}$, Huang Qin-huang ${ }^{2, b}$ \\ 1 Zhaber field road No.3519, Shanghai, China, 200436 \\ 2. 3. Fu'zhou Fujian China, 350000) \\ E-mail: ${ }^{a}$ greetree_1234@163.com, ${ }^{b}$ fengsheng_1234@163.com
}

Key words: Complex electromagnetic environment; Radar signal sorting; Software sorting algorithm

\begin{abstract}
This article analyzes the current electronic warfare is faced with numerous and complex electromagnetic environment, in the dual machine cooperative reconnaissance system, the reconnaissance receiver with a timely and accurate manner to select the application requirements of the radar emitter signal, the use of the software sorting algorithm, through simulation, the performance of the algorithm can meet the requirements of the system.
\end{abstract}

\section{Introduction}

Electronic warfare is an important means of attack and defense in the modern war[1,2]. With the rapid development of radar technology, the electromagnetic environment is very complex, the reconnaissance receiver may face hundreds or even thousands of radiation sources, from the high density signal environment for signal separation is the important part of the information war[3].

In cooperative reconnaissance system, it is important to determine the characteristics and geographical distribution of radar signal in a timely and accurate manner, and to determine the use of radar emitter, the weapon system and threat level, which lead us to make timely, accurate and effective response.

In this paper, the basic concept of radar signal sorting technology is introduced, and the composition of the software is introduced in detail. Finally, the signal sorting algorithm is studied and simulated in detail, which shows that the algorithm has good separation effect.

\section{2 overview of radar signal sorting}

Radar signal sorting[4] is a key component of the electronic warfare. The modern radar is developed in many functions and multi - purpose, and it is often used to design a variety of complex waveforms. At the same time, the density of the pulse current can reach $200000 / \mathrm{sec}, 2000000 /$ sec.

Radar signal sorting[5,6] is a reconnaissance receiver receives dense overlapping pulse flow, from these random overlapping pulse flow in isolated from each radar pulse train and select the useful signal. At present, the radar signal sorting is based on pulse carrier frequency, pulse arrival direction, pulse width and pulse description words information to preprocess the pulse characteristic parameters such as RF, PA, PW, and direction of arrival (DOA) and will be the radar signal sorting processing.

The processing of radar signal sorting is shown in Figure 1. The reconnaissance receiver receives signals from the complex signal environment, completes the formation and organization of the pulse, then extracts and identifies the signal with the local source signal. 


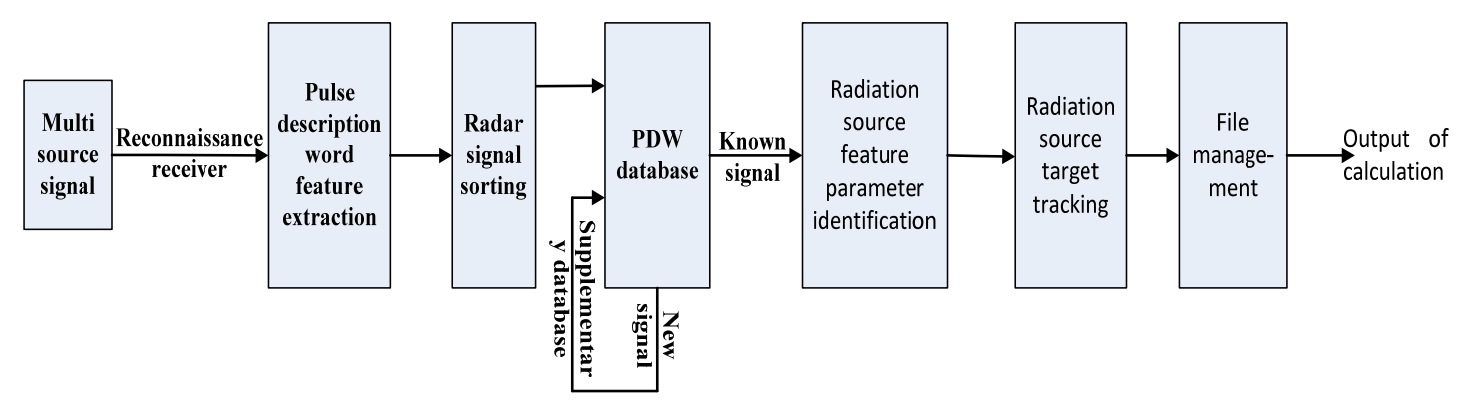

Figure 1 Process of Radar Signal Sorting

\section{3 software sorting}

In this design, the software is used to sort the radar signal. The overall structure of the sorting process is shown in Figure 2. Multi parameter radar signal sorting is mainly composed of three parts, signal pre sorting, main sorting and back-end processing.

Radar signal sorting including known radiation source matching and deduction, the purpose is to reduce the signal density, the random pulse flow random overlapping, according to certain criteria, cluster according to the orientation of the signal parameters, carrier frequency and pulse width, pulse flow dilution, for sorting the next starting point; the second step is the main sorting, clustering in pulse on time, pulse clustering difference between multilevel statistical difference, find out the frequency parameters of possible values, then according to the frequency parameter values to find all of the clustering, pulse, extraction, extraction, according to the number of a certain threshold, confirm whether the corresponding signal under the condition of frequency is established, finally the same parameter identification radar. The third step is the follow-up processing, including the statistical analysis of the data, the deduction of false radiation sources, the correlation processing, the threat level discrimination and the updated radar database.

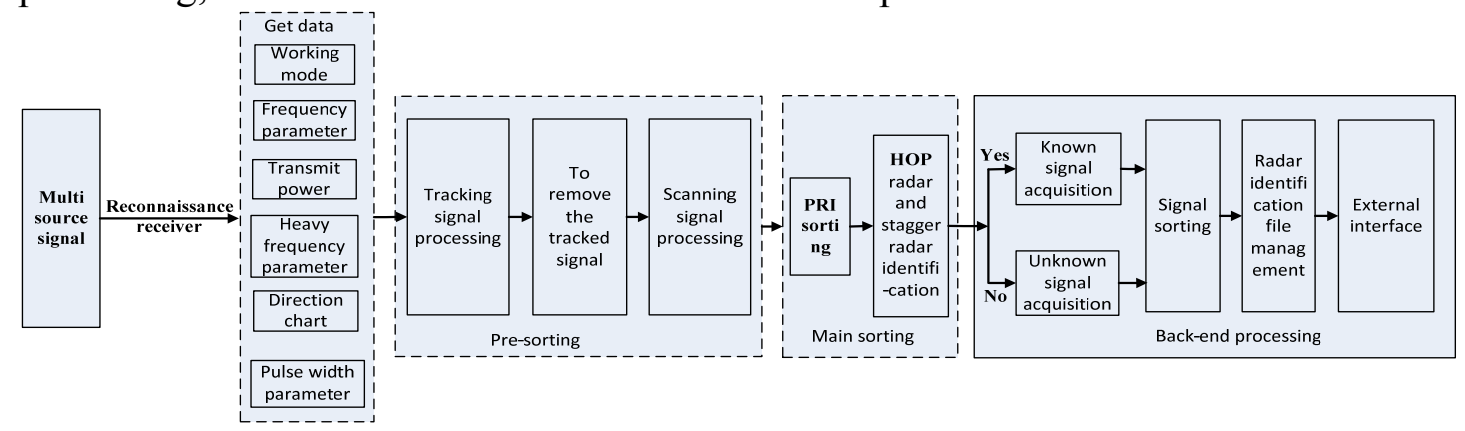

Figure 2 overall block diagram of software

\section{4 algorithm and analysis}

\section{1 sequence difference square graph sorting algorithm}

Software sorting and processing algorithm[7,8] in the engineering trade-offs, the main letter in the sorting process speed and the accuracy of the first processing results. In this design, we use Difference Histogram Sequential (SDIF) sorting algorithm, and the algorithm flow chart is shown in Figure 3. Based on the statistical rule of pulse repetition interval, the possible repetition interval value is found out, then the signal pulse is extracted, and the possible radar signal is confirmed. The specific process is as follows:

According to the difference between the of Arrive Time (TOA) of two adjacent pulses, the difference of the first level difference histogram is formed, the threshold is calculated and then the sub harmonic test is carried out to determine whether the PRI can be searched. If we can successfully isolate the corresponding sequence, from the sampling sequence is deducted and remaining pulse sequence from the first level began to form a new difference histogram, after the sub harmonic test, if more than a peak over threshold, from over the threshold peak corresponding to the small pulse interval sequence search and finally staggered differential. 


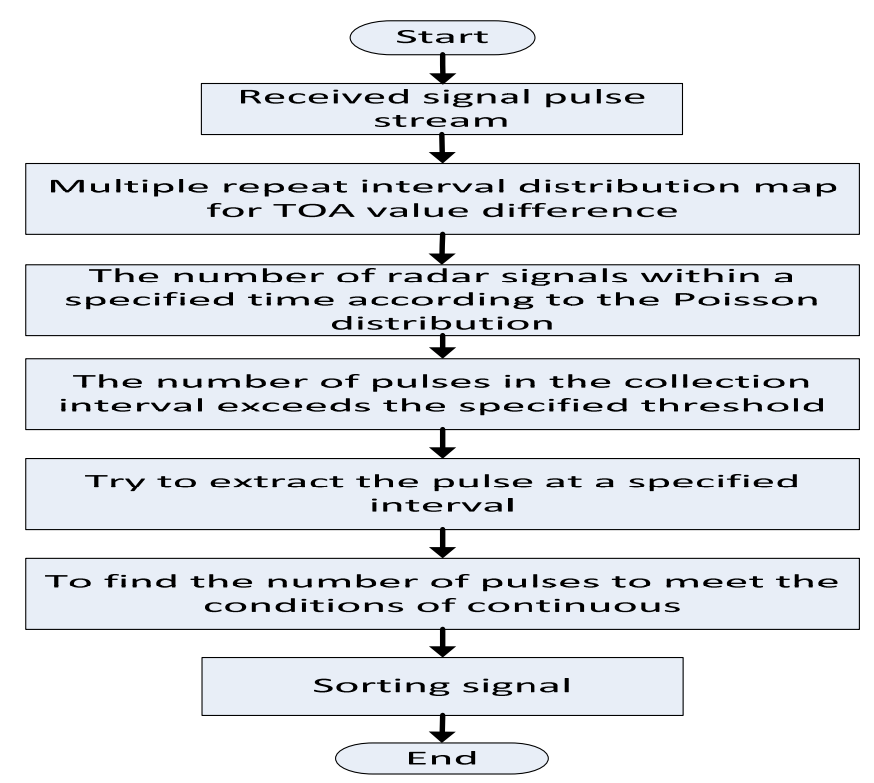

Figure 3 The Process of SDIF Arithmetic

The detection threshold is represented by the formula (1), and its value is an important part of the SDIF algorithm.

$$
P(\tau)=\frac{x E}{\tau}
$$

$x$ is less than 1 constant, $E$ is the total number of input pulses, $\tau$ is the pulse interval. The formula shows that the threshold value is proportional to the total number of input pulses, and the $E$ is inversely proportional to the pulse interval $\tau$.

When the radar exist at the same time, the input pulse number and adjacent pulse interval can be as random events, obeys the Poisson distribution, the limited observation time $T$ points to $n$ pulse punch interval, in time interval $\left(\tau=t_{2}-t_{1}\right.$ with $k$ stochastic Poisson probability for:

$$
P_{k}(\tau)=\frac{(\lambda x)^{k}}{k !} e^{-\lambda \tau} \quad k=0,1, \cdots
$$

Where $\lambda=n / T$, which represents the number of pulses within a unit time interval. The probability approximation for the adjacent two pulse interval $\tau$ is:

$$
P_{0}(\tau)=e^{-\lambda \tau}
$$

Type (3) is a general form of the first order difference histogram, since the histogram is actually an approximate value of the probability distribution function of a random event, so the higher difference histogram is in the form of an exponential distribution. The number of pulse groups constituting the difference histogram of the $C$ level is $(E-C)$, that is, the $(E-C)$ event occurs in the time, and the parameter $\lambda=1 / \mu N$, which is the best detection threshold function is obtained:

$$
T_{\text {threshold }}(\tau)=x(E-C) e^{-\tau / \mu N}
$$

Among them, $E$ is the total number of pulses, the $C$ is the difference histogram of the series, $\mu$ is less than 1 of the normal number, $N$ is the maximum value of the histogram statistics. $x$ constant is determined by experiments.

\section{2 simulation analysis}

In this case the simulation analysis and sorting of radar signal is overlap burst in together, the radar pulse repetition interval respectively, $P R I_{1}=280 \mu \mathrm{s}, P R I_{2}=400 \mu \mathrm{s}, \quad P R I_{3}=530 \mu \mathrm{s}$, jitter amount is $1 \%$, pulse for a total of 450 , SDIF threshold parameters: $x=0.4, k=0.3$ 。

The simulation results are shown in Figure 4 (a), (b), (c) and (d), respectively, for the first order to four order difference histograms. 


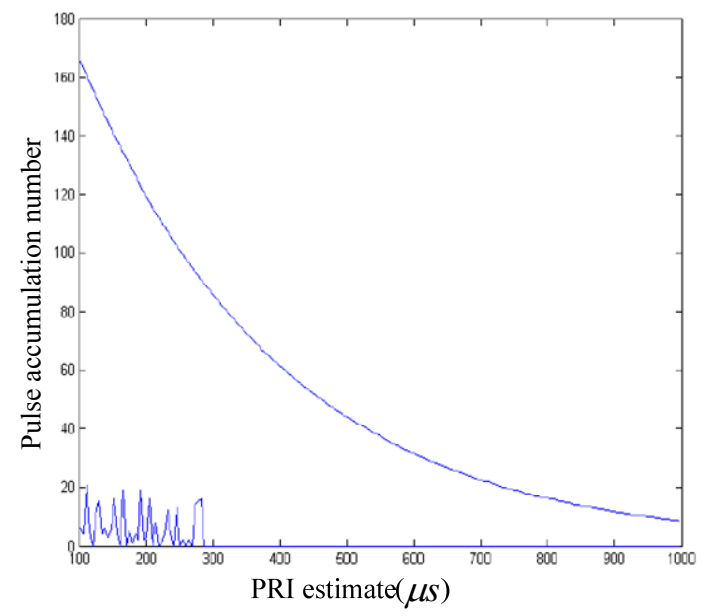

(a) First order SDIF simulation results

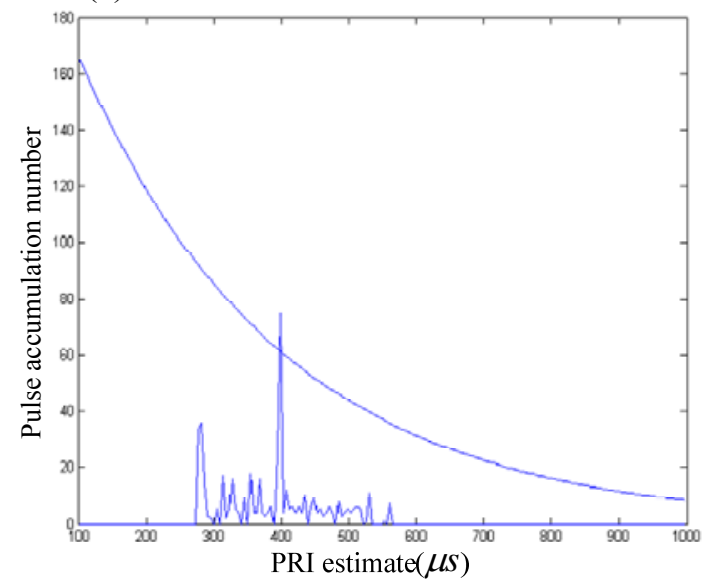

(c) Three order SDIF simulation results

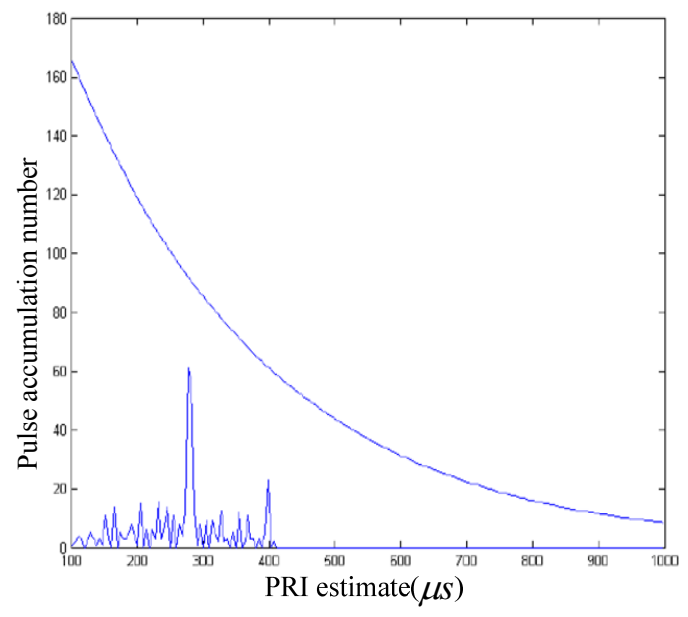

(b) Two order SDIF simulation results

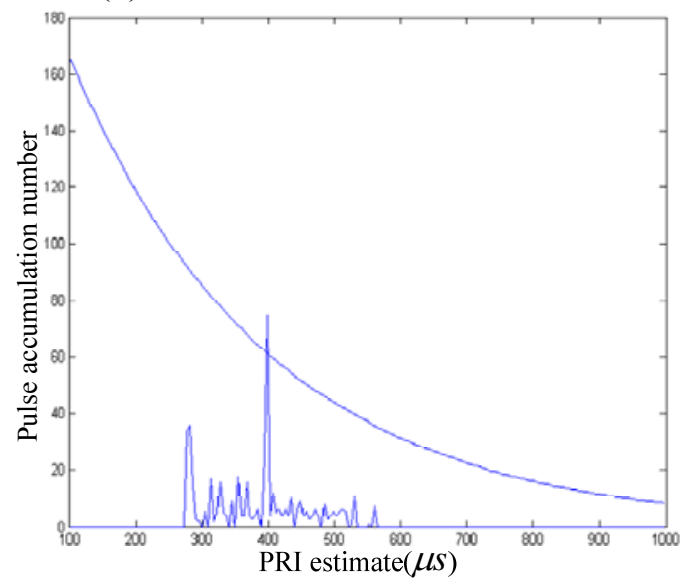

(d) Four order SDIF simulation results

Figure 4 simulation results

From the simulation results, we can see that the SDIF algorithm is less than 5\%, the PRI jitter is less than $3 \%$ of the radar pulse, as long as the threshold parameters are chosen properly, the algorithm can achieve good accuracy, and can meet the requirements of the system's signal sorting.

5 conclusions

With the rapid development of radar technology, signal sorting in electronic warfare becomes difficult, and the amount of the radiation source is increasing rapidly. The signal form is more and more complex and diverse. In this paper, based on the task requirement, the SDIF algorithm is studied. The performance of the system is verified by simulation analysis. It has good estimation accuracy and can meet the requirement of signal sorting in this system.

\section{Reference}

[1] Zhao Guoqing (Second Edition) [M]. Xi'an: Electronic Science and Technology University Press, 2012.6.

[2] Wang Jiegui, Jin Xueming. Overview of modern radar signal sorting technology [J]. radar science and technology, 2006 (2):105-107.

[3] Zou Shun. Radar signal sorting and fine feature analysis [D]. Xi'an: Northwestern Polytechnical University, 2006

[4] ANN, Li Yunzhen. Performance analysis of PRI detection of pulse radar signal [J]. modern radar, 2007, 29 (2):35-37.

Mandia H. K. Digital Signal Processing for Radar Recognition in Dense Radar Envinonments[D]. Electrical Engineering Department Leeds, University 1988:32-155., [5]

Cai Jun Gao, Jin-ming Zhao, Jian-min. Application of FPGA and CPLD in the radar signal sorting processor[J]. Systems Engineering and Electronics. [6] 2001, 23 (10): 22-24. 
$\mathrm{Hu}$ Hong-ying Kang Jing et, Li-na Guan, al. Instantaneous frequency estimation based on empirical mode decomposition. Proceedings of the 7th World Congress on Intelligent Control and Chongqing Automation, June:, China, 2008, 25-27. [7]

Zhang G X Hu, L Z Jin, W D. Resemblance coefficient based intrapulse feature extraction approach for radar emitter signals[J]. Chinese Journal of Electronics [8], 14, 2005 (2): 337-341. 